\title{
Constructing Sibson Elements for a Rectangular Mesh
}

\section{E. Gilisinn}

U. S. DEPARTMENT OF COMMERCE

Technology Adminıstration

Mathematics and Computational Sciences Division National Institute of Standards and Technology

Gaithersburg, MD 20899-8910

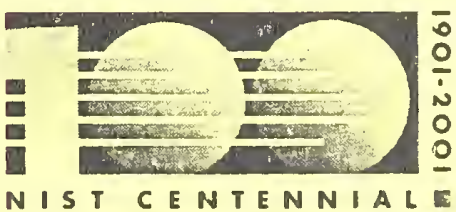





\section{Constructing Sibson Elements for a Rectangular Mesh}

D. E. Gilsinn

U. S. DEPARTMENT OF COMMERCE

Technology Adminıstration

Mathematics and Computational Sciences Division

National Institute of Standards

and Technology

Gaıthersburg, MD 20899-8910

February 28, 2001

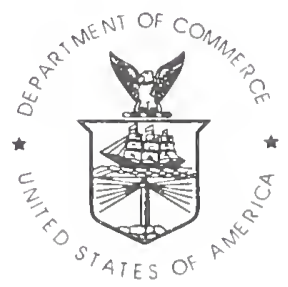

U.S. DEPARTMENT OF COMMERCE Donald L. Evans, Secretary

NATIONAL INSTITUTE OF STANDARDS AND TECHNOLOGY

Dr. Karen H. Brown, Actıng Director 



\title{
Constructing Sibson Elements for a Rectangular Mesh
}

\author{
David E. Gilsinn \\ Mathematics and Computational Sciences Division \\ National Institute of Standards and Technology \\ 100 Bureau Drive, Stop 8910 \\ Gaithersburg, MD 20899-8910
}

March 2, 2001

\begin{abstract}
This paper documents the construction of a finite element, called the Sibson element. The shape function of this element is formed on rectangular grids by $C^{1}$ splines defined on a triangulation of each subrectangle by dividing it into four subtriangles formed by drawing the diagonals. The splines are constructed from bivariate cubic polynomials $z(x, y)$ and are written in such a way that they are linear functions of the values $z, \partial z / \partial x, \partial z / \partial y$ at each node of the rectangle with bivariate polynomial coefficients up to order three. Conditions are given for the existence of such an element. They are used to construct the bivariate polynomial coefficients, first for a unit rectangle and then for a general rectangle. Since the first and second derivatives of these functions are sometimes needed they are also given.
\end{abstract}

Key Words: bivariate polynomial; rectangular mesh; shape function; Sibson element; spline; triangulation

\section{Introduction}

When modeling urban and rural terrain data we are usually given points $\left(x_{2}, y_{i}, z_{i}\right)$ for $i=1,2, \cdots$, not necessarily uniformly spaced. We would like to construct a $C^{1}$ function to interpolate the given data. Lawson $[5,6]$ has pioneered $C^{1}$ interpolation over triangular grids. Han and Schumaker [1] interpolate data on rectangular grids by $C^{1}$ splines defined on a triangulation of each subrectangle by dividing it into four subtriangles formed by drawing the diagonals. This subdivision is called the Sibson split. They then construct the $C^{1}$ spline by using a Bernstein-Bézier form. Recently Lavery $[3,4]$ has used the Sibson split on rectangular grids of terrain data but has constructed $C^{1}$ splines over these Sibson elements directly from bivariate cubic polynomials. He briefly describes the derivation in Lavery [4] but only gives the final formula for the spline on one of the subtriangles of a subrectangle. It is the object of this report to present the details involved with the derivation of these bivariate cubic elements.

We begin with a tensor-product grid with nodes $x_{i}$, for $i=0,1, \cdots, I$, and $y_{j}$, for $j=0,1, \cdots, J$, that form strictly monotonic partitions of the finite real intervals $\left[x_{0}, x_{I}\right]$ and $\left[y_{0}, y_{J}\right]$ respectively. The nodes need not be uniformly spaced. The cubic splines $z(x, y)$ will consist of $C^{1}$ smooth, piecewise cubic Sibson elements on the tensor-product grid. We also suppose that we are given, by some means, elevation and derivative values $z_{i j}, z_{i j}^{x}=\partial z / \partial x, z_{i j}^{y}=\partial z / \partial y$ at each node.

To create a Sibson element on a rectangle $\left(x_{i}, x_{i+1}\right) \times\left(y_{j}, y_{j+1}\right)$ we first divide the rectangle into four triangles by drawing the two diagonals of the rectangle. The Sibson element is a shape function, $z(x, y)$, that is cubic within each triangle, is $C^{1}$ on the lines separating the four triangles, has $\partial z / \partial x$ that is linear along the edges $x=x_{i}$ and $x=x_{i+1}$ of the rectangle and has derivative $\partial z / \partial y$ that is linear along the edges $y=y_{j}$ and $y=y_{j+1}$. 
The report is structured as follows. Section 2 describes the specific sufficient conditions for constructing a Sibson element on a square with unit length on each side. The necessary equations for determining the bivariate polynomials in each of the four Sibson triangles of the unit element are derived in Section 3. Solving for the bivariate polynomial equations is done in Section 4. In Section 5 the coeffficients are written in terms of the three items of information at each of the corner nodes. In Section 6 the final Sibson element shape functions are constructed for the unit element. In Section 7 the Sibson element shape functions on the unit square are transformed to shape functions on an arbitrary scaled rectangle. Finally, in Section 8 the first and second derivatives of the Sibson element splines are computed.

\section{Defining the Conditions for Constructing a Unit Sibson Element}

In this section we develop the Sibson element on a reference unit square in order to simplify the construction. In a later section we will introduce a transformation that will map this element to non-uniform Sibson elements. Figure 1 shows a unit square with positive r-axis pointing downwards and positive s-axis pointing to the right. The corners are identified as $(0,0),(1,0),(1,1),(0,1)$. The diagonal line from $(0,0)$ to $(1,1)$ is given by the equation $s=r$ and that from $(1,0)$ to $(0,1)$ is given by $s=r-1$. The triangles are labeled $0,1,2,3$, beginning with the triangle identified by the points $(0,0),(1,0),(1 / 2,1 / 2)$, and proceeding in a counterclockwise manner.

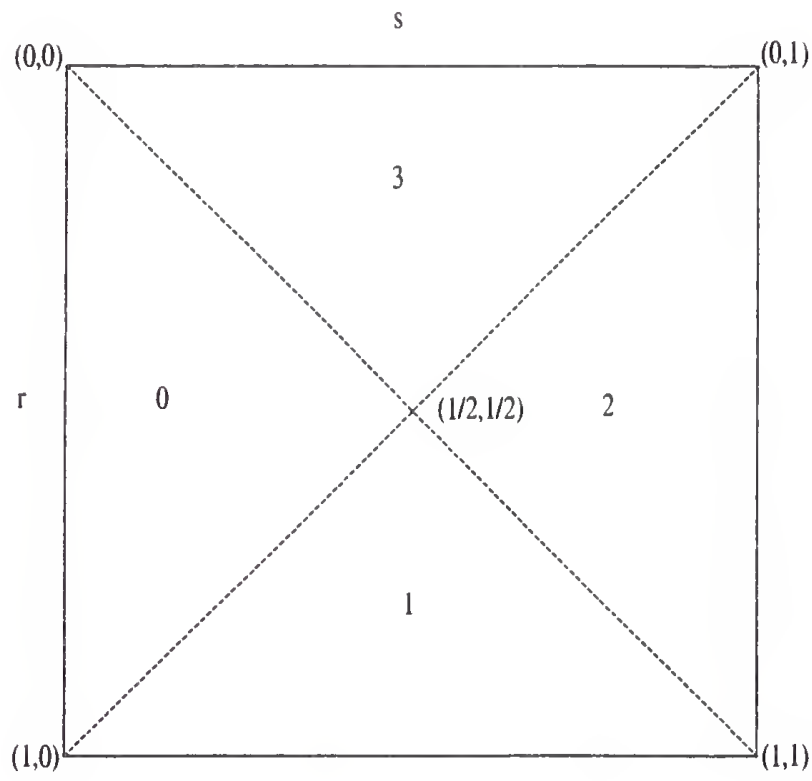

Figure 1: Unit Sibson Element

Cubic polynomials are defined on each triangle in the following two forms. First, for triangles indexed by $k=0, \cdots, 3$ the bivariate polynomials are written as

$$
\begin{aligned}
z_{k}(r, s)= & c_{30}^{k} r^{3}+c_{21}^{k} r^{2} s+c_{12}^{k} r s^{2}+c_{03}^{k} s^{3} \\
& +c_{20}^{k} r^{2}+c_{11}^{k} r s+c_{02}^{k} s^{2}+c_{10}^{k} r
\end{aligned}
$$




$$
+c_{01}^{k} s+c_{00}^{k}
$$

and for triangles indexed by $k=1,2$ they are written as

$$
\begin{aligned}
z_{k}(r, s)= & c_{30}^{k}(1-r)^{3}+c_{21}^{k}(1-r)^{2}(1-s)+c_{12}^{k}(1-r)(1-s)^{2}+c_{03}^{k}(1-s)^{3} \\
& +c_{20}^{k}(1-r)^{2}+c_{11}^{k}(1-r)(1-s)+c_{02}^{k}(1-s)^{2}+c_{10}^{k}(1-r) \\
& +c_{01}^{k}(1-s)+c_{00}^{k} .
\end{aligned}
$$

These bivariate polynomial forms are selected so that a point $(r, s)$ in triangle 0 is matched with a symmetric point $((1-r),(1-s))$ in triangle 2 along a line through the point $(1 / 2,1 / 2)$. A similar symmetry holds for triangles 1 and 3 . The coefficients $c_{p q}^{k}$ are indexed as follows. The superscript $k$ indexes the triangle and the subscript $p q$ represents the exponentials in the term $c_{p q}^{k} r^{p} s^{q}$ or $c_{p q}^{k}(1-r)^{p}(1-s)^{q}$.

These four bivariate cubic polynomials must interpolate at the four corners, must have $\frac{\partial z}{\partial x}$ that is linear along the edges $x=x_{i}$ and $x=x_{i+1}$ of the rectangle and have derivative $\partial z / \partial y$ that is linear along the edges $y=y_{j}$ and $y=y_{j+1}$ and satisfy the $C^{1}$ conditions across the diagonals. The interpolation conditions at the corners are given by the following identities.

$$
\begin{aligned}
z_{0}(0,0) & =z_{3}(0,0) \\
\frac{\partial z_{0}}{\partial r}(0,0) & =\frac{\partial z_{3}}{\partial r}(0,0) \\
\frac{\partial z_{0}}{\partial s}(0,0) & =\frac{\partial z_{3}}{\partial s}(0,0) \\
z_{0}(1,0) & =z_{1}(1,0) \\
\frac{\partial z_{0}}{\partial r}(1,0) & =\frac{\partial z_{1}}{\partial r}(1,0) \\
\frac{\partial z_{0}}{\partial s}(1,0) & =\frac{\partial z_{1}}{\partial s}(1,0) \\
z_{2}(0,1) & =z_{3}(0,1) \\
\frac{\partial z_{2}}{\partial r}(0,1) & =\frac{\partial z_{3}}{\partial r}(0,1) \\
\frac{\partial z_{2}}{\partial s}(0,1) & =\frac{\partial z_{3}}{\partial s}(0,1) \\
z_{1}(1,1) & =z_{2}(1,1) \\
\frac{\partial z_{1}}{\partial r}(1,1) & =\frac{\partial z_{2}}{\partial r}(1,1) \\
\frac{\partial z_{1}}{\partial s}(1,1) & =\frac{\partial z_{2}}{\partial s}(1,1)
\end{aligned}
$$

In the unit cell case, the outer boundary conditions imply that the following partial derivatives, for $r \in$ $[0,1], s \in[0,1]$,

$$
\begin{aligned}
& \frac{\partial z_{0}}{\partial s}(r, 0) \\
& \frac{\partial z_{1}}{\partial r}(1, s) \\
& \frac{\partial z_{2}}{\partial s}(r, 1) \\
& \frac{\partial z_{3}}{\partial r}(0, s)
\end{aligned}
$$

are linear. Finally, the polynomials must be $C^{1}$ across the diagonals. Between triangles 0 and 3 , along the line $s=r$, the following equations must be satisfied.

$$
z_{0}(r, r)=z_{3}(r, r),
$$




$$
\begin{aligned}
& \frac{\partial z_{0}}{\partial r}(r, r)=\frac{\partial z_{3}}{\partial r}(r, r), \\
& \frac{\partial z_{0}}{\partial s}(r, r)=\frac{\partial z_{3}}{\partial s}(r, r) .
\end{aligned}
$$

Between triangles 1 and 2, along the line $s=r$,

$$
\begin{aligned}
z_{1}(r, r) & =z_{2}(r, r), \\
\frac{\partial z_{1}}{\partial r}(r, r) & =\frac{\partial z_{2}}{\partial r}(r, r), \\
\frac{\partial z_{1}}{\partial s}(r, r) & =\frac{\partial z_{2}}{\partial s}(r, r) .
\end{aligned}
$$

Between triangles 0 and 1 , along the line $s=1-r$,

$$
\begin{aligned}
z_{0}(r, 1-r) & =z_{1}(r, 1-r), \\
\frac{\partial z_{0}}{\partial r}(r, 1-r) & =\frac{\partial z_{1}}{\partial r}(r, 1-r), \\
\frac{\partial z_{0}}{\partial s}(r, 1-r) & =\frac{\partial z_{1}}{\partial s}(r, 1-r) .
\end{aligned}
$$

Finally, between triangles 2 and 3 , along the line $s=1-r$,

$$
\begin{aligned}
z_{2}(r, 1-r) & =z_{3}(r, 1-r), \\
\frac{\partial z_{2}}{\partial r}(r, 1-r) & =\frac{\partial z_{3}}{\partial r}(r, 1-r), \\
\frac{\partial z_{2}}{\partial s}(r, 1-r) & =\frac{\partial z_{3}}{\partial s}(r, 1-r) .
\end{aligned}
$$

All of these conditions will produce the necessary equations that must be satisfied by the forty unknown coefficients. In the next section we will develop these equations.

\section{Necessary Equations for Coefficient Determination}

The equations developed in this section are all determined by the conditions in the previous section. They arise from simply inserting the appropriate polynomial, (1) or (2), into either the interpolation, boundary or diagonal conditions and equating appropriate coefficients. In this section $z_{i j}$ represents the elevation data at the corner $(i, j)$ of a cell. $z_{i j}^{r}$ is the derivative with respect to $r$ at $(i, j)$ and $z_{i j}^{s}$ is the derivative with respect to $s$ at $(i, j)$.

We first develop the equations that arise from the interpolation conditions. We will use (3) to develop the coefficient relations that arise as a result of the interpolation at the unit cell corners. In particular, at $(0,0)$ simple substitutions show that

$$
\begin{aligned}
& c_{00}^{0}=z_{00}, \\
& c_{00}^{3}=z_{00}, \\
& c_{10}^{0}=z_{00}^{r}, \\
& c_{10}^{3}=z_{00}^{r}, \\
& c_{01}^{0}=z_{00}^{s}, \\
& c_{01}^{3}=z_{00}^{s} .
\end{aligned}
$$

At $(1,0)$ the coefficient relations are given by

$$
c_{30}^{0}+c_{20}^{0}+c_{10}^{0}+c_{00}^{0}=z_{10}
$$




$$
\begin{aligned}
c_{03}^{1}+c_{02}^{1}+c_{01}^{1}+c_{00}^{1} & =z_{10} \\
3 c_{30}^{0}+2 c_{20}^{0}+c_{10}^{0} & =z_{10}^{r} \\
-c_{12}^{1}-c_{11}^{1}-c_{10}^{1} & =z_{10}^{r} \\
c_{21}^{0}+c_{11}^{0}+c_{01}^{0} & =z_{10}^{s} \\
-3 c_{03}^{1}-2 c_{02}^{1}-c_{01}^{1} & =z_{10}^{s}
\end{aligned}
$$

At $(0,1)$ the relations are

$$
\begin{aligned}
c_{30}^{2}+c_{20}^{2}+c_{10}^{2}+c_{00}^{2} & =z_{01} \\
c_{03}^{3}+c_{02}^{3}+c_{01}^{3}+c_{00}^{3} & =z_{01} \\
-3 c_{30}^{2}-2 c_{20}^{2}-c_{10}^{2} & =z_{01}^{r} \\
c_{12}^{3}+c_{11}^{3}+c_{10}^{3} & =z_{01}^{r} \\
-c_{21}^{2}-c_{11}^{2}-c_{01}^{2} & =z_{01}^{s} \\
3 c_{03}^{3}+2 c_{02}^{3}+c_{01}^{3} & =z_{01}^{s}
\end{aligned}
$$

The last interpolation relations, given at $(1,1)$, are

$$
\begin{aligned}
& c_{00}^{1}=z_{11} \\
& c_{00}^{2}=z_{11} \\
& c_{10}^{1}=-z_{11}^{r} \\
& c_{10}^{2}=-z_{11}^{r} \\
& c_{01}^{1}=-z_{11}^{s} \\
& c_{01}^{2}=-z_{11}^{s}
\end{aligned}
$$

At the external boundaries of the cells $\partial z / \partial x$ is linear along the edges $x=x_{i}$ and $x=x_{i+1}$ of the rectangle and the derivative $\partial z / \partial y$ is linear along the edges $y=y_{j}$ and $y=y_{\jmath+1}$. By differentiating (1) and (2), evaluating them on the appropriate boundaries and setting the quadratic coefficients to zero it is easy to see that

$$
\begin{aligned}
& c_{21}^{0}=0 \\
& c_{12}^{1}=0 \\
& c_{21}^{2}=0 \\
& c_{12}^{3}=0
\end{aligned}
$$

The $C^{1}$ conditions along the diagonals are obtained by simply evaluating the relations $(5),(6),(7),(8)$, and equating the coefficients of like powers. Therefore, along $s=r$ between triangle 0 and 3 we obtain the equations

$$
\begin{aligned}
c_{30}^{0}+c_{21}^{0}+c_{12}^{0}+c_{03}^{0} & =c_{30}^{3}+c_{21}^{3}+c_{12}^{3}+c_{03}^{3} \\
c_{20}^{0}+c_{11}^{0}+c_{02}^{0} & =c_{20}^{3}+c_{11}^{3}+c_{02}^{3} \\
c_{10}^{0}+c_{01}^{0} & =c_{10}^{3}+c_{01}^{3} \\
c_{00}^{0} & =c_{00}^{3} \\
3 c_{30}^{0}+2 c_{21}^{0}+c_{12}^{0} & =3 c_{30}^{3}+2 c_{21}^{3}+c_{12}^{3} \\
2 c_{20}^{0}+c_{11}^{0} & =2 c_{20}^{3}+c_{11}^{3} \\
c_{10}^{0} & =c_{10}^{3} \\
c_{21}^{0}+2 c_{12}^{0}+3 c_{03}^{0} & =c_{21}^{3}+2 c_{12}^{3}+3 c_{03}^{3} \\
c_{11}^{0}+2 c_{02}^{0} & =c_{11}^{3}+2 c_{02}^{3} \\
c_{01}^{0} & =c_{01}^{3}
\end{aligned}
$$


Along the line $s=r$, between triangles 1 and 2, we obtain

$$
\begin{aligned}
& c_{30}^{1}+c_{21}^{1}+c_{12}^{1}+c_{03}^{1}= c_{30}^{2}+c_{21}^{2}+c_{12}^{2}+c_{03}^{2} \\
& 3 c_{30}^{1}+3 c_{21}^{1}+3 c_{12}^{1}+3 c_{03}^{1} \\
&+c_{20}^{1}+c_{11}^{1}+c_{02}^{1}= 3 c_{30}^{2}+3 c_{21}^{2}+3 c_{12}^{2} \\
&+3 c_{03}^{2}+c_{20}^{2}+c_{11}^{2}+c_{02}^{2} \\
&-3 c_{30}^{1}-3 c_{21}^{1}-3 c_{12}^{1}-3 c_{03}^{1}-2 c_{20}^{1}=-3 c_{30}^{2}-3 c_{21}^{2}-3 c_{12}^{2}-3 c_{03}^{2} \\
&-2 c_{11}^{1}-2 c_{02}^{1}-c_{10}^{1}-c_{01}^{1}=-2 c_{20}^{2}-2 c_{11}^{2}-2 c_{02}^{2}-c_{10}^{2}-c_{01}^{2} \\
& \\
& c_{30}^{1}+c_{21}^{1}+c_{12}^{1}+c_{03}^{1}+c_{20}^{1}+c_{11}^{1}= c_{30}^{2}+c_{21}^{2}+c_{12}^{2}+c_{03}^{2} \\
&+c_{02}^{1}+c_{10}^{1}+c_{01}^{1}+c_{00}^{1}=+c_{20}^{2}+c_{11}^{2}+c_{02}^{2}+c_{10}^{2}+c_{01}^{2}+c_{00}^{2} \\
&-3 c_{30}^{1}-2 c_{21}^{1}-c_{12}^{1}=2 c_{21}^{2}-c_{12}^{2} \\
&-3 c_{30}^{1}-2 c_{21}^{1}-c_{12}^{1}-2 c_{20}^{1}-c_{11}^{1}-c_{10}^{1}=-3 c_{30}^{2}-2 c_{21}^{2}-c_{12}^{2}-2 c_{20}^{2}-c_{11}^{2}-c_{10}^{2} \\
&-c_{21}^{1}-2 c_{12}^{1}-3 c_{03}^{1}=-c_{21}^{2}-2 c_{12}^{2}-3 c_{03}^{2} \\
& 2 c_{21}^{1}+4 c_{12}^{1}+6 c_{03}^{1}+c_{11}^{1}+2 c_{02}^{1}= 2 c_{21}^{2}+4 c_{12}^{2}+6 c_{03}^{2}+c_{11}^{2}+2 c_{02}^{2} \\
&-c_{21}^{1}-2 c_{12}^{1}-3 c_{03}^{1}-c_{11}^{1}-2 c_{02}^{1}-c_{01}^{1}=-c_{21}^{2}-2 c_{12}^{2}-3 c_{03}^{2}-c_{11}^{2}-2 c_{02}^{2}-c_{01}^{2}
\end{aligned}
$$

Along the line $s=1-r$, between triangles 0 and 1 , we obtain

$$
\begin{aligned}
c_{30}^{0}-c_{21}^{0}+c_{12}^{0}-c_{03}^{0} & =-c_{30}^{1}+c_{21}^{1}-c_{12}^{1}+c_{03}^{1} \\
c_{21}^{0}-2 c_{12}^{0}+3 c_{03}^{0}+c_{20}^{0}-c_{11}^{0}+c_{02}^{0} & =3 c_{30}^{1}-2 c_{21}^{1}+c_{12}^{1}+c_{20}^{1}-c_{11}^{1}+c_{02}^{1} \\
c_{12}^{0}-3 c_{03}^{0}+c_{11}^{0}-2 c_{02}^{0}+c_{10}^{0}-c_{01}^{0} & =-3 c_{30}^{1}+c_{21}^{1}-2 c_{20}^{1}+c_{11}^{1}-c_{10}^{1}+c_{01}^{1} \\
c_{03}^{0}+c_{02}^{0}+c_{01}^{0}+c_{00}^{0} & =c_{30}^{1}+c_{20}^{1}+c_{10}^{1}+c_{00}^{1} \\
3 c_{30}^{0}-2 c_{21}^{0}+c_{12}^{0} & =-3 c_{30}^{1}+2 c_{21}^{1}-c_{12}^{1} \\
2 c_{21}^{0}-2 c_{12}^{0}+2 c_{20}^{0}-c_{11}^{0} & =6 c_{30}^{1}-2 c_{21}^{1}+2 c_{20}^{1}-c_{11}^{1} \\
c_{12}^{0}+c_{11}^{0}+c_{10}^{0} & =-3 c_{30}^{1}-2 c_{20}^{1}-c_{10}^{1} \\
c_{21}^{0}-2 c_{12}^{0}+3 c_{03}^{0} & =-c_{21}^{1}+2 c_{12}^{1}-3 c_{03}^{1} \\
2 c_{12}^{0}-6 c_{03}^{0}+c_{11}^{0}-2 c_{02}^{0} & =2 c_{21}^{1}-2 c_{12}^{1}+c_{11}^{1}-2 c_{02}^{1} \\
3 c_{03}^{0}+2 c_{02}^{0}+c_{01}^{0} & =-c_{21}^{1}-c_{11}^{1}-c_{01}^{1}
\end{aligned}
$$

Finally, along the line $s=1-r$, between triangles 2 and 3 , we obtain

$$
\begin{aligned}
-c_{30}^{2}+c_{21}^{2}-c_{12}^{2}+c_{03}^{2} & =c_{30}^{3}-c_{21}^{3}+c_{12}^{3}-c_{03}^{3} \\
3 c_{30}^{2}-2 c_{21}^{2}+c_{12}^{2}+c_{20}^{2}-c_{11}^{2}+c_{02}^{2} & =c_{21}^{3}-2 c_{12}^{3}+3 c_{03}^{3}+c_{20}^{3}-c_{11}^{3}+c_{02}^{3} \\
-3 c_{30}^{2}+c_{21}^{2}-2 c_{20}^{2}+c_{11}^{2}-c_{10}^{2}+c_{01}^{2} & =c_{12}^{3}-3 c_{03}^{3}+c_{11}^{3}-2 c_{02}^{3}+c_{10}^{3}-c_{01}^{3} \\
c_{30}^{2}+c_{20}^{2}+c_{10}^{2}+c_{00}^{2} & =c_{03}^{3}+c_{02}^{3}+c_{01}^{3}+c_{00}^{3} \\
-3 c_{30}^{2}+2 c_{21}^{2}-c_{12}^{2} & =3 c_{30}^{3}-2 c_{21}^{3}+c_{12}^{3} \\
6 c_{30}^{2}-2 c_{21}^{2}+2 c_{20}^{2}-c_{11}^{2} & =2 c_{21}^{3}-2 c_{12}^{3}-c_{11}^{3}+2 c_{20}^{3} \\
-3 c_{30}^{2}-2 c_{20}^{2}-c_{10}^{2} & =c_{12}^{3}+c_{11}^{3}+c_{10}^{3} \\
-c_{21}^{2}+2 c_{12}^{2}-3 c_{03}^{2} & =c_{21}^{3}-2 c_{12}^{3}+3 c_{03}^{3} \\
2 c_{21}^{2}-2 c_{12}^{2}+c_{11}^{2}-2 c_{02}^{2} & =2 c_{12}^{3}-6 c_{03}^{3}+c_{11}^{3}-2 c_{02}^{3} \\
-c_{21}^{2}-c_{11}^{2}-c_{01}^{2} & =3 c_{03}^{3}+2 c_{02}^{3}+c_{01}^{3}
\end{aligned}
$$




\section{Finding a Solution}

There are sixty eight equations developed in the previous section, from (9) to (76), but only forty unknowns, so we expect twenty eight redundant equations. Of the forty unknowns sixteen are immediately determined from equations (9) through (32) and (27) through (36). With these given, it is necessary to find the other twenty four solvable equations and test that the rest of the equations are redundant. Some redundancies are obvious. For example, equation (39) is redundant with (29) through (32), whereas other redundancies are not so clear.

The first two equations that can be solved are (15) and (17) for $c_{20}^{0}$ and $c_{30}^{0}$ in the form

$$
\begin{aligned}
& c_{20}^{0}=3 z_{10}-z_{10}^{r}-2 c_{10}^{0}-3 c_{00}^{0} \\
& c_{30}^{0}=z_{10}-c_{20}^{0}-c_{20}^{0}-c_{10}^{0}-c_{00}^{0}
\end{aligned}
$$

where $c_{00}^{0}$ and $c_{10}^{0}$ are known from equations (9) and (11) respectively. The solutions given in this section will be developed in terms of coefficients with either known values or previously computed values. The representation of the coefficients in terms of the data at the four corners of the cell will be done in the next section. That step of the process required the assistance of a symbolic manipulation program.

The second two equations that can be solved are (16) and (20) for $c_{02}^{1}$ and $c_{03}^{1}$ as

$$
\begin{aligned}
& c_{02}^{1}=3 z_{10}+z_{10}^{s}-2 c_{01}^{1}-3 c_{00}^{1} \\
& c_{03}^{1}=z_{10}-c_{02}^{1}-c_{01}^{1}-c_{00}^{1} .
\end{aligned}
$$

The next two equations that can be solved are (21) and (23) for $c_{20}^{2}$ and $c_{30}^{2}$ as

$$
\begin{aligned}
& c_{20}^{2}=3 z_{01}+z_{01}^{r}-2 c_{10}^{2}-3 c_{00}^{2} \\
& c_{30}^{2}=z_{01}-c_{20}^{2}-c_{10}^{2}-c_{00}^{2} .
\end{aligned}
$$

From (22) and (20) we can solve for $c_{02}^{3}$ and $c_{03}^{3}$ as

$$
\begin{aligned}
& c_{02}^{3}=3 z_{01}-z_{01}^{s}-2 c_{01}^{3}-3 c_{00}^{3} \\
& c_{03}^{3}=z_{01}-c_{02}^{3}-c_{01}^{3}-c_{00}^{3} .
\end{aligned}
$$

Now we solve (19), (18), (25), and (24) for $c_{11}^{0}, c_{11}^{1}, c_{11}^{2}$, and $c_{11}^{3}$ respectively as

$$
\begin{aligned}
c_{11}^{0} & =z_{10}^{s}-c_{21}^{0}-c_{01}^{0} \\
c_{11}^{1} & =-z_{10}^{r}-c_{12}^{1}-c_{10}^{1} \\
c_{11}^{2} & =-x_{01}^{s}-c_{21}^{2}-c_{01}^{2} \\
c_{11}^{3} & =z_{01}^{r}-c_{12}^{3}-c_{10}^{3}
\end{aligned}
$$

Since $c_{20}^{0}, c_{11}^{0}$, and $c_{11}^{3}$ have been computed we can solve (42) for $c_{20}^{3}$ as

$$
c_{20}^{3}=c_{20}^{0}+\frac{1}{2} c_{11}^{0}-\frac{1}{2} c_{11}^{3} .
$$

Again, since $c_{11}^{3}, c_{02}^{3}$, and $c_{11}^{0}$ have been computed we can solve (45) for $c_{02}^{0}$ as

$$
c_{02}^{0}=\frac{1}{2} c_{11}^{3}+c_{02}^{3}-\frac{1}{2} c_{11}^{0} .
$$

We can now solve (72) for $c_{21}^{3}$ as

$$
c_{21}^{3}=\frac{1}{2}\left(6 c_{30}^{2}-2 c_{21}^{2}+2 c_{20}^{2}-c_{11}^{2}+2 c_{12}^{3}+c_{11}^{3}-2 c_{20}^{3}\right)
$$


To solve for $c_{20}^{1}$ insert (51) into (53) and cancel like terms in (53) to get

$$
c_{20}^{1}=\frac{1}{2}\left(-c_{11}^{1}-c_{10}^{1}+2 c_{20}^{2}+c_{11}^{2}+c_{10}^{2}\right)
$$

To solve for $c_{02}^{2}$, multiply (47) by 3 , insert the result into (49) and cancel like terms in (49) to get

$$
c_{02}^{2}=\frac{1}{2}\left(2 c_{20}^{1}+2 c_{11}^{1}+2 c_{02}^{1}+c_{10}^{1}+c_{01}^{1}-2 c_{20}^{2}-2 c_{11}^{2}-c_{10}^{2}-c_{01}^{2}\right)
$$

To solve for $c_{12}^{0}$, first solve (66) for $c_{21}^{1}$ in terms of known values and the unknown $c_{03}^{0}$, which will be computed later, to get

$$
c_{21}^{1}=-c_{11}^{1}-c_{01}^{1}-3 c_{03}^{0}-2 c_{02}^{0}-c_{01}^{0} .
$$

This is a temporary equation for $c_{21}^{1}$ that will be used for substitution into (64). Once this substitution is made, terms for $c_{03}^{0}$ cancel in equation (64). The final result is

$$
c_{12}^{0}=\frac{1}{2}\left(c_{21}^{0}-c_{11}^{1}-c_{01}^{1}-2 c_{02}^{0}-c_{01}^{0}-2 c_{12}^{1}+3 c_{03}^{1}\right)
$$

where all of the terms on the right are known.

From (63) solve for $c_{30}^{1}$ as

$$
c_{30}^{1}=\frac{1}{3}\left(-2 c_{20}^{1}-c_{10}^{1}-c_{12}^{0}-c_{11}^{0}-c_{10}\right)
$$

From (61) solve for $c_{21}^{1}$ as

$$
c_{21}^{1}=\frac{1}{2}\left(3 c_{30}-2 c_{21}^{0}+c_{12}^{0}+3 c_{30}^{1}+c_{12}^{1}\right)
$$

From (66) we can now solve for $c_{03}^{0}$ as

$$
c_{03}^{0}=\frac{1}{3}\left(-c_{21}^{1}-c_{11}^{1}-c_{01}^{1}-2 c_{02}^{0}-c_{01}^{0}\right) .
$$

From (41) solve for $c_{30}^{3}$ as

$$
c_{30}^{3}=\frac{1}{3}\left(3 c_{30}^{0}+2 c_{21}^{0}+c_{12}^{0}-2 c_{21}^{3}-c_{12}^{3}\right) .
$$

From (53) solve for $c_{12}^{2}$ as

$$
\begin{aligned}
c_{12}^{2}= & 3 c_{30}^{1}+2 c_{21}^{1}+c_{12}^{1}+2 c_{20}^{1}+c_{11}^{1}+c_{10}^{1} \\
& -3 c_{30}^{2}-2 c_{21}^{2}-2 c_{20}^{2}-c_{11}^{2}-c_{10}^{2}
\end{aligned}
$$

Finally, from (54) we can solve for $c_{03}^{2}$ as

$$
c_{03}^{2}=\frac{1}{3}\left(c_{21}^{1}+2 c_{12}^{1}+3 c_{03}^{1}-c_{21}^{2}-2 c_{12}^{2}\right) .
$$

As is clear these coefficients were solved using a subset of the sixty eight equations. All of the other equations are redundant. This has been verified by a symbolic manipulation program. 


\section{Representing the Coefficients in Terms of Corner Data}

The coefficients can now be expanded in terms of the twelve data items at the corners of the unit square. This again is done by a symbolic manipulation program. The results are given below.

$$
\begin{aligned}
& c_{00}^{0}=z_{00} \\
& c_{00}^{3}=z_{00} \\
& c_{10}^{0}=z_{00}^{r} \\
& c_{01}^{0}=z_{00}^{s} \\
& c_{10}^{3}=z_{00}^{r} \\
& c_{01}^{3}=z_{00}^{s} \\
& c_{00}^{1}=z_{11} \\
& c_{00}^{2}=z_{11} \\
& c_{10}^{1}=-z_{11}^{r} \\
& c_{01}^{1}=-z_{11}^{s} \\
& c_{10}^{2}=-z_{11}^{r} \\
& c_{01}^{2}=-z_{11}^{s} \\
& c_{21}^{0}=0 \\
& c_{12}^{1}=0 \\
& c_{21}^{2}=0 \\
& c_{12}^{3}=0 \\
& c_{20}^{0}=3 z_{10}-z_{10}^{r}-2 z_{00}^{r}-3 z_{00} \\
& c_{30}^{0}=-2 z_{10}+z_{00}^{r}+2 z_{00}+z_{10}^{r} \\
& c_{02}^{1}=3 z_{10}+z_{10}^{s}+2 z_{11}^{s}-3 z_{11} \\
& c_{03}^{1}=-2 z_{10}-z_{10}^{s}-z_{11}^{s}+2 z_{11} \\
& c_{20}^{2}=3 z_{01}+z_{01}^{r}+2 z_{11}^{T}-3 z_{11} \\
& c_{30}^{2}=-2 z_{01}-z_{01}^{r}-z_{11}^{r}+2 z_{11} \\
& c_{02}^{3}=3 z_{01}-z_{01}^{s}-2 z_{00}^{s}-3 z_{00} \\
& c_{03}^{3}=-2 z_{01}+z_{01}^{s}+z_{00}^{s}+2 z_{00} \\
& c_{11}^{0}=z_{10}^{s}-z_{00}^{s} \\
& c_{11}^{1}=z_{11}^{r}-z_{10}^{r} \\
& c_{11}^{2}=z_{11}^{s}-z_{01}^{s} \\
& c_{11}^{3}=z_{01}^{r}-z_{00}^{r} \\
& c_{20}^{3}=3 z_{10}-z_{10}^{r}-\frac{3}{2} z_{00}^{r}-3 z_{00}+\frac{1}{2} z_{10}^{s}-\frac{1}{2} z_{00}^{s}-\frac{1}{2} z_{01}^{r} \\
& c_{02}^{0}=\frac{1}{2} z_{01}^{r}-\frac{1}{2} z_{00}^{r}+3 z_{01}-z_{01}^{s}-\frac{3}{2} z_{00}^{s}-3 z_{00}-\frac{1}{2} z_{10}^{s} \\
& c_{21}^{3}=-3 z_{01}-z_{01}^{r}-z_{11}^{r}+3 z_{11}-\frac{1}{2} z_{11}^{s}+\frac{1}{2} z_{01}^{s}+z_{00}^{r} \\
& -3 z_{10}+z_{10}^{r}+3 z_{00}-\frac{1}{2} z_{10}^{s}+\frac{1}{2} z_{00}^{s} \\
& c_{20}^{1}=\frac{3}{2} z_{11}^{r}+\frac{1}{2} z_{10}^{r}+3 z_{01}+z_{01}^{r}-3 z_{11}+\frac{1}{2} z_{11}^{s}-\frac{1}{2} z_{01}^{s} \\
& c_{02}^{2}=\frac{1}{2} z_{11}^{r}-\frac{1}{2} z_{10}^{r}-3 z_{11}+\frac{3}{2} z_{11}^{s}+\frac{1}{2} z_{01}^{s}+3 z_{10}+z_{10}^{s}
\end{aligned}
$$




$$
\begin{aligned}
c_{12}^{0}= & -\frac{1}{2} z_{11}^{r}+\frac{1}{2} z_{10}^{r}-z_{11}^{s}-\frac{1}{2} z_{01}^{r}+\frac{1}{2} z_{00}^{r}-3 z_{01} \\
& +z_{01}^{s}+z_{00}^{s}+3 z_{00}-z_{10}^{s}-3 z_{10}+3 z_{11} \\
c_{30}^{1}= & -\frac{1}{2} z_{11}^{r}-\frac{1}{2} z_{10}^{r}-z_{01}-\frac{1}{2} z_{01}^{r}+z_{11}-\frac{1}{2} z_{00}^{r}-z_{00}+z_{10} \\
c_{21}^{1}= & -3 z_{01}-z_{01}^{r}-z_{11}^{r}+3 z_{11}-\frac{1}{2} z_{11}^{s}+\frac{1}{2} z_{01}^{s} \\
& +z_{00}^{r}-3 z_{10}+z_{10}^{r}+3 z_{00}-\frac{1}{2} z_{10}^{s}+\frac{1}{2} z_{00}^{s} \\
c_{03}^{0}= & -z_{01}-z_{11}+\frac{1}{2} z_{11}^{s}+\frac{1}{2} z_{01}^{s}+z_{10}+z_{00}+\frac{1}{2} z_{10}^{s}+\frac{1}{2} z_{00}^{s} \\
c_{30}^{3}= & z_{00}+\frac{1}{2} z_{00}^{r}-z_{11}+\frac{1}{2} z_{11}^{r}-z_{10}+\frac{1}{2} z_{10}^{r}+z_{01}+\frac{1}{2} z_{01}^{r} \\
c_{12}^{2}= & -\frac{1}{2} z_{11}^{r}+\frac{1}{2} z_{10}^{r}-z_{11}^{s}-\frac{1}{2} z_{01}^{r}+\frac{1}{2} z_{00}^{r}-3 z_{01} \\
& +z_{01}^{s}+z_{00}^{s}+3 z_{00}-z_{10}^{s}-3 z_{10}+3 z_{11} \\
c_{03}^{2}= & -z 00-\frac{1}{2} z_{00}^{s}+z_{11}-\frac{1}{2} z_{11}^{s}-z_{10} \\
& -\frac{1}{2} z_{10}^{s}+z_{01}-\frac{1}{2} z_{01}^{s} \\
& -z{ }^{2}
\end{aligned}
$$

\section{Sibson Element Shape Functions on a Unit Cell}

In this section we introduce the coefficients given in (95) into the appropriate polynomials (1) or (2) given in Section 2. We will then rewrite the polynomials in the form of shape functions where the coefficients of the twelve corner data items are polynomials, whose order does not exceed three. This is done by simply rearranging the spatial and corner data in the polynomials (1) and (2) after substituting (95). The Sibson element shape functions for each of the four triangles are given by

$$
\begin{aligned}
z_{0}(r, s)= & \left(1-3 s^{2}-3 r^{2}+s^{3}+2 r^{3}+3 r s^{2}\right) z_{00}+\left(r^{3}+\frac{1}{2} r s^{2}-2 r^{2}-\frac{1}{2} s^{2}+r\right) z_{00}^{r} \\
& +\left(r s^{2}+\frac{1}{2} s^{3}-r s-\frac{3}{2} s^{2}+s\right) z_{00}^{s}+\left(-2 r^{3}-3 r s^{2}+s^{3}+3 r^{2}\right) z_{10} \\
& +\left(r^{3}+\frac{1}{2} r s^{2}-r^{2}\right) z_{10}^{r}+\left(-r s^{2}+\frac{1}{2} s^{3}+r s-\frac{1}{2} s^{2}\right) z_{10}^{s} \\
& +\left(-3 r s^{2}-s^{3}+3 s^{2}\right) z_{01}+\left(-\frac{1}{2} r s^{2}+\frac{1}{2} s^{2}\right) z_{01}^{r}+\left(\frac{1}{2} s^{3}-s^{2}+r s^{2}\right) z_{01}^{s} \\
& +\left(3 r s^{2}-s^{3}\right) z_{11}+\left(-\frac{1}{2} r s^{2}\right) z_{11}^{r}+\left(-r s^{2}+\frac{1}{2} s^{3}\right) z_{11}^{s} \\
z_{1}(r, s)= & \left(-(1-r)^{3}+3(1-r)^{2}(1-s)\right) z_{00}+\left(-\frac{1}{2}(1-r)^{3}+(1-r)^{2}(1-s)\right) z_{00}^{r} \\
& +\left(\frac{1}{2}(1-r)^{2}(1-s)\right) z_{00}^{s}+\left((1-r)^{3}-3(1-r)^{2}(1-s)-2(1-s)^{3}+3(1-s)^{2}\right) z_{10} \\
& +\left(-\frac{1}{2}(1-r)^{3}+(1-r)^{2}(1-s)+\frac{1}{2}(1-r)^{2}-(1-r)(1-s)\right) z_{10}^{r} \\
& +\left(-\frac{1}{2}(1-r)^{2}(1-s)-(1-s)^{3}+(1-s)^{2}\right) z_{10}^{s} \\
& +\left(-(1-r)^{3}-3(1-r)^{2}(1-s)+3(1-r)^{2}\right) z_{01}
\end{aligned}
$$




$$
\begin{aligned}
& +\left(-\frac{1}{2}(1-r)^{2}-(1-r)^{2}(1-s)+(1-r)^{2}\right) z_{01}^{r}+\left(\frac{1}{2}(1-r)^{2}(1-s)-\frac{1}{2}(1-r)^{2}\right) z_{01}^{s} \\
& +\left((1-r)^{3}+3(1-r)^{2}(1-s)+2(1-s)^{3}-3(1-r)^{2}-3(1-s)^{2}+1\right) z_{11} \\
& +\left(-\frac{1}{2}(1-r)^{3}-(1-r)^{2}(1-s)+\frac{3}{2}(1-r)^{2}+(1-r)(1-s)-(1-r)\right) z_{11}^{r} \\
& +\left(-\frac{1}{2}(1-r)^{2}(1-s)-(1-s)^{3}+\frac{1}{2}(1-r)^{2}+2(1-s)^{2}-(1-s)\right) z_{11}^{s} \\
& z_{2}(r, s)=\left(3(1-r)(1-s)^{2}-(1-s)^{3}\right) z_{00}+\left(\frac{1}{2}(1-r)(1-s)^{2}\right) z_{00}^{r} \\
& +\left(\frac{1}{2}(1-r)(1-s)^{2}-\frac{1}{2}(1-s)^{3}\right) z_{00}^{s}+\left(-3(1-r)(1-s)^{2}-(1-s)^{3}+3(1-s)^{2}\right) z_{10} \\
& +\left(\frac{1}{2}(1-r)(1-s)^{2}-\frac{1}{2}(1-s)^{2}\right) z_{10}^{r}+\left(-(1-r)(1-s)^{2}-\frac{1}{2}(1-s)^{3}+(1-s)^{2}\right) z_{10}^{s} \\
& +\left(-2(1-r)^{3}-3(1-r)(1-s)^{2}+(1-s)^{3}+3(1-r)^{2}\right) z_{01} \\
& +\left(-(1-r)^{3}-\frac{1}{2}(1-r)(1-s)^{2}+(1-r)^{2}\right) z_{01}^{r} \\
& +\left((1-r)(1-s)^{2}-\frac{1}{2}(1-s)^{2}-(1-r)(1-s)+\frac{1}{2}(1-s)^{2}\right) z_{01}^{s} \\
& +\left(2(1-r)^{3}+3(1-r)(1-s)^{2}+(1-s)^{3}-3(1-r)^{2}-3(1-s)^{2}+1\right) z_{11} \\
& +\left(-(1-r)^{3}-\frac{1}{2}(1-r)(1-s)^{2}+2(1-r)^{2}+\frac{1}{2}(1-s)^{2}-(1-r)\right) z_{11}^{r} \\
& +\left(-(1-r)(1-s)^{2}-\frac{1}{2}(1-s)^{3}+(1-r)(1-s)+\frac{3}{2}(1-s)^{2}-(1-s)\right) z_{11}^{s} \\
& z_{3}(r, s)=\left(r^{3}+3 r^{2} s+2 s^{3}-3 r^{2}-3 s^{2}+1\right) z_{00}+\left(\frac{1}{2} r^{3}+r^{2} s-\frac{3}{2} r^{2}-r s+r\right) z_{00}^{r} \\
& +\left(\frac{1}{2} r^{2} s+r^{3}-\frac{1}{2} r^{2}-2 s^{2}+s\right) z_{00}^{s}+\left(-r^{3}-3 r^{2} s+3 r^{2}\right) z_{10} \\
& +\left(\frac{1}{2} r^{3}+r^{2} s-r^{2}\right) z_{10}^{r}+\left(-\frac{1}{2} r^{2} s+\frac{1}{2} r^{2}\right) z_{10}^{s} \\
& +\left(r^{3}-3 r^{2} s-2 s^{3}+3 s^{2}\right) z_{01}+\left(\frac{1}{2} r^{3}-r^{2} s-\frac{1}{2} r^{2}+r s\right) z_{01}^{r} \\
& +\left(\frac{1}{2} r^{2} s+s^{3}-s^{2}\right) z_{01}^{s}+\left(-r^{3}+3 r^{2} s\right) z_{11}+\left(\frac{1}{2} r^{3}-r^{2} s\right) z_{11}^{r}+\left(-\frac{1}{2} r^{2} s\right) z_{11}^{s}
\end{aligned}
$$

\section{Shape Functions on a Scaled Grid}

In the previous sections we constructed the Sibson element shape functions on a unit square. In this section we will extend this construction to rectangular grids with uniform spacings along the $x$ and $y$ axes. The Sibson split for this grid spacing is given in Figure 2. The spacings, although uniform along each axis, need not be the same for the $x$ and $y$ axes. In particular, let $\left(x_{i}, x_{i+1}\right) \times\left(y_{j}, y_{j+1}\right)$ be a subrectangle in the overall mesh. Since mesh spacings need not be uniform, let $\Delta x_{i}=x_{i+1}-x_{i}$ and $\Delta y_{j}=y_{j+1}-y_{j}$ Now we define

$$
r=\frac{x-x_{i}}{\Delta x_{i}}
$$




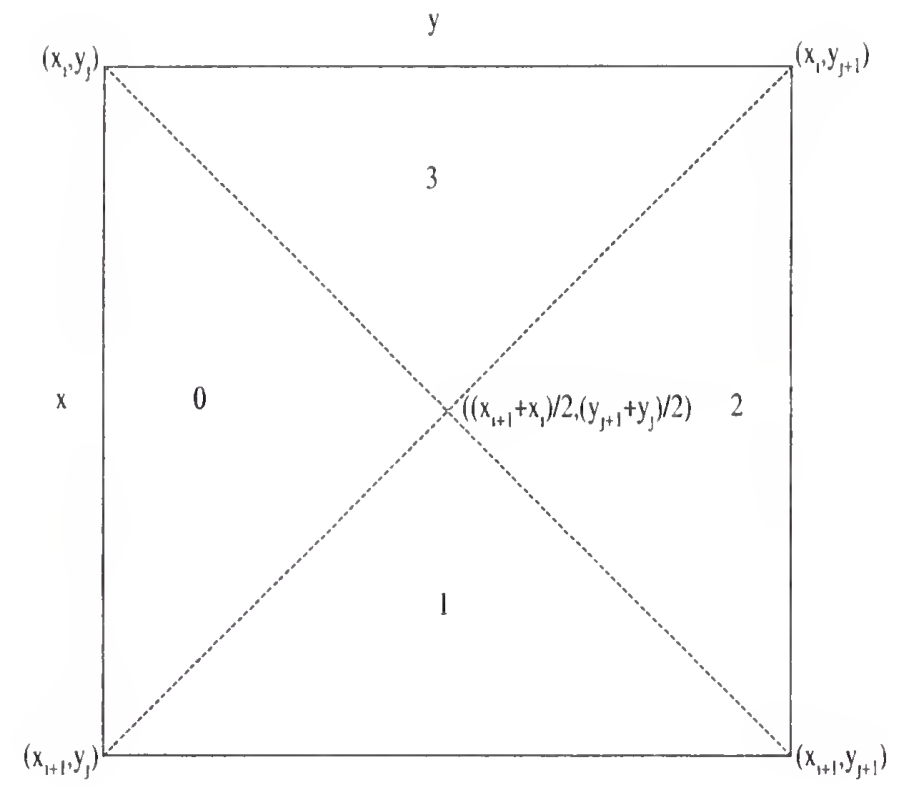

Figure 2: Rectangular Sibson Element

$$
s=\frac{y-y_{j}}{\Delta y_{\jmath}}
$$

where $x_{i} \leq x \leq x_{\imath+1}$ and $y_{j} \leq y_{j+1}$. Then

$$
\begin{aligned}
& \frac{\partial x}{\partial r}=\Delta x_{i} \\
& \frac{\partial y}{\partial s}=\Delta y_{\jmath}
\end{aligned}
$$

and

$$
\begin{aligned}
& z_{00}^{r}=\frac{\partial z}{\partial r}=\frac{\partial z}{\partial x}\left(x_{i}, y_{j}\right) \frac{\partial x}{\partial r}=\Delta x_{\imath} z_{i j}^{x} \\
& z_{00}^{s}=\frac{\partial z}{\partial s}=\frac{\partial z}{\partial y}\left(x_{i}, y_{j}\right) \frac{\partial y}{\partial s}=\Delta y_{j} z_{i j}^{s} .
\end{aligned}
$$

By a similar argument

$$
\begin{aligned}
& z_{10}^{r}=\Delta x_{i} z_{i+1, j}^{x} \\
& z_{10}^{s}=\Delta y_{j} z_{i+1, j}^{y} \\
& z_{01}^{r}=\Delta x_{i} z_{i, j+1}^{x} \\
& z_{01}^{s}=\Delta y_{j} z_{i, j+1}^{y} \\
& z_{11}^{r}=\Delta x_{i} z_{i+1, j+1}^{x} \\
& z_{11}^{s}=\Delta y_{j} z_{i+1, j+1}^{y} .
\end{aligned}
$$


Also

$$
\begin{aligned}
& \begin{array}{l}
z(10) \quad=1,1 \\
z 11 \quad=2+1,1
\end{array} \\
& \text { zil } \quad=1,1+1 \\
& \approx 11 \ldots=21+1,1+1
\end{aligned}
$$

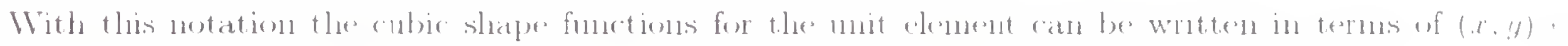
$\left(x_{2}, x_{2}+1\right) \times\left(y_{j}, y_{j}+1\right)$, wherer and s are detined by (10)

$$
\begin{aligned}
& z_{0}(x, y)=\left(1-3 s^{2}-3 r^{2}+s^{3}+2 r^{3}+3 r s^{2}\right) z_{3}+\Delta r \cdot\left(r^{3}+\frac{1}{2} r s^{2}-2 r^{2}-\frac{1}{2} s^{2}+r\right) z^{2} \\
& +\Delta y_{3}\left(r s^{2}+\frac{1}{2} s^{3}-r s-\frac{3}{2} s^{2}+s\right) z^{4}+\left(-2 r^{3}-3 r s^{2}+s^{3}+3 r^{2}\right)+2+1.3 \\
& +\Delta r_{2}\left(r^{3}+\frac{1}{2} r s^{2}-r^{2}\right) z_{1}+1,3+\Delta y_{3}\left(-r s^{2}+\frac{1}{2} s^{3}+r s^{2}-\frac{1}{2} s^{2}\right) z^{y}+1, j \\
& +\left(-3 r s^{2}-s^{3}+3 r^{2}\right) z_{2, j+1}+\Delta r,\left(-\frac{1}{2} r s^{2}+\frac{1}{2} s^{2}\right) z_{i, j+1}^{r} \\
& +\Delta y_{3}\left(\frac{1}{2} s^{3}-s^{2}+r^{2}\right)=\frac{y}{2, j+1}+\left(3 r r^{2}-s^{3}\right) z+1, j+1 \\
& +\Delta x_{2}\left(-\frac{1}{2} r r^{2}\right) z^{2}+1+1+1+\Delta y_{3}\left(-r s^{2}+\frac{1}{2} s^{3}\right)=z^{4}+1, j+1
\end{aligned}
$$

$z_{1}(x, y) \quad\left(-(1-r)^{3}+3(1-r)^{2}(1-s)\right) z_{2,1}$

$$
\begin{aligned}
& +\Delta r_{2}\left(-\frac{1}{2}(1-r)^{3}+(1-r)^{2}(1-s)\right)=z_{i j}^{x}+\Delta y_{1}\left(\frac{1}{2}(1-r)^{2}(1-s)\right)=z_{i j}^{4} \\
& +\left((1-r)^{3}-3(1-r)^{2}(1-s)-2(1-s)^{3}+3(1-s)^{2}\right) z i+1, s \\
& +\Delta r_{2}\left(-\frac{1}{2}(1 \quad r)^{3}+(1-r)^{2}(1-s)+\frac{1}{2}(1-r)^{2}-(1-r)(1-s)\right) 2 i+1, r \\
& +\Delta y_{1}\left(-\frac{1}{2}(1-r)^{2}(1-s)-(1-s)^{3}+(1-s)^{2}\right) z_{i+1} \\
& +\left(-(1-r)^{3}-3(1-r)^{2}(1-s)+3(1-r)^{2}\right) z_{2, j+1} \\
& +\Delta x_{2}\left(-\frac{1}{2}(1-r)^{2}-(1-r)^{2}(1-s)+(1-r)^{2}\right) z_{2, J+1}^{x} \\
& +\Delta y_{j}\left(\frac{1}{2}(1-r)^{2}(1-s) \quad \frac{1}{2}(1-r)^{2}\right)=z_{i, 1+1}^{4} \\
& +\left((1-r)^{3}+3(1-r)^{2}(1-s)+2(1-s)^{3}-3(1-r)^{2} \quad 3(1-s)^{2}+1\right) z_{1-1}+1 \\
& +\Delta x_{1}\left(-\frac{1}{2}(1 \quad r)^{3}-(1-r)^{2}(1-s)+\frac{3}{2}(1-r)^{2}+(1 \quad r)(1 \quad s)(1 \quad r)\right)=i+1+1
\end{aligned}
$$

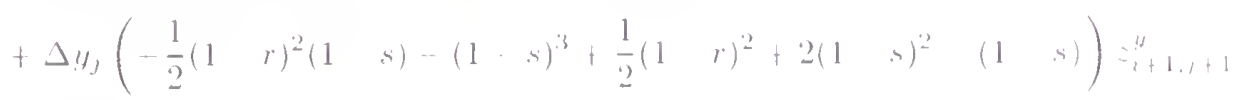

$$
\begin{aligned}
& z_{2}(x, y)=\left(3(1-r)(1-s)^{2} \cdots(1-s)^{3}\right) z_{2}+\Delta r_{1}\left(\frac{1}{2}(1+r)(1 \quad s)^{2}\right) z_{1}^{3} \\
& +\Delta y_{3}\left(\frac{1}{2}(1-r)(1-s)^{2}-\frac{1}{2}(1-s)^{3}\right)=4
\end{aligned}
$$




$$
\begin{aligned}
& +\left(-3(1-r)(1-s)^{2}-(1-s)^{3}+3(1-s)^{2}\right) z_{i+1, j} \\
& +\Delta x_{i}\left(\frac{1}{2}(1-r)(1-s)^{2}-\frac{1}{2}(1-s)^{2}\right) z_{i+1, j}^{x} \\
& +\Delta y_{j}\left(-(1-r)(1-s)^{2}-\frac{1}{2}(1-s)^{3}+(1-s)^{2}\right) z_{i+1, j}^{y} \\
& +\left(-2(1-r)^{3}-3(1-r)(1-s)^{2}+(1-s)^{3}+3(1-r)^{2}\right) z_{i, j+1} \\
& +\Delta x_{i}\left(-(1-r)^{3}-\frac{1}{2}(1-r)(1-s)^{2}+(1-r)^{2}\right) z_{i, j+1}^{x} \\
& +\Delta y_{j}\left((1-r)(1-s)^{2}-\frac{1}{2}(1-s)^{2}-(1-r)(1-s)+\frac{1}{2}(1-s)^{2}\right) z_{i, j+1}^{y} \\
& +\left(2(1-r)^{3}+3(1-r)(1-s)^{2}+(1-s)^{3}-3(1-r)^{2}-3(1-s)^{2}+1\right) z_{i+1, j+1} \\
& +\Delta x_{i}\left(-(1-r)^{3}-\frac{1}{2}(1-r)(1-s)^{2}+2(1-r)^{2}+\frac{1}{2}(1-s)^{2}-(1-r)\right) z_{i+1, j+1}^{x} \\
& +\Delta y_{j}\left(-(1-r)(1-s)^{2}-\frac{1}{2}(1-s)^{3}+(1-r)(1-s)+\frac{3}{2}(1-s)^{2}-(1-s)\right) z_{i+1, j+1}^{y} \\
& z_{3}(x, y)=\left(r^{3}+3 r^{2} s+2 s^{3}-3 r^{2}-3 s^{2}+1\right) z_{i j}+\Delta x_{i}\left(\frac{1}{2} r^{3}+r^{2} s-\frac{3}{2} r^{2}-r s+r\right) z_{i j}^{x} \\
& +\Delta y_{j}\left(\frac{1}{2} r^{2} s+r^{3}-\frac{1}{2} r^{2}-2 s^{2}+s\right) z_{i j}^{y}+\left(-r^{3}-3 r^{2} s+3 r^{2}\right) z_{i+1, j} \\
& +\Delta x_{i}\left(\frac{1}{2} r^{3}+r^{2} s-r^{2}\right) z_{i+1, j}^{x}+\Delta y_{\jmath}\left(-\frac{1}{2} r^{2} s+\frac{1}{2} r^{2}\right) z_{i+1, j}^{y} \\
& +\left(r^{3}-3 r^{2} s-2 s^{3}+3 s^{2}\right) z_{i, 3+1}+\Delta x_{i}\left(\frac{1}{2} r^{3}-r^{2} s-\frac{1}{2} r^{2}+r s\right) z_{i, j+1}^{x} \\
& +\Delta y_{j}\left(\frac{1}{2} r^{2} s+s^{3}-s^{2}\right) z_{i, j+1}^{y}+\left(-r^{3}+3 r^{2} s\right) z_{i+1, j+1} \\
& +\Delta x_{i}\left(\frac{1}{2} r^{3}-r^{2} s\right) z_{i+1, j+1}^{x}+\Delta y_{j}\left(-\frac{1}{2} r^{2} s\right) z_{i+1, j+1}^{y}
\end{aligned}
$$

\section{Derivatives}

Since Lavery [4] uses the first and second derivatives of the Sibson element splines in his interpolation and approximation procedures, we will include both sets of derivatives in this section for completeness. Again we will use the notation

$$
\begin{aligned}
r & =\frac{x-x_{i}}{\Delta x_{i}} \\
s & =\frac{y-y_{j}}{\Delta y_{\jmath}}
\end{aligned}
$$

and $\Delta x=x_{i+1}-x_{i}, \Delta y=y_{j+1}-y_{j}$. Then

$$
\begin{aligned}
& \frac{\partial r}{\partial x}=\frac{1}{\Delta x_{i}} \\
& \frac{\partial s}{\partial y}=\frac{1}{\Delta y_{j}}
\end{aligned}
$$




\subsection{First Derivatives}

From the cubic splines for each of the triangles in a cell, given by (105), (106), (107), and (108), we can apply the chain rule and (110) to compute the first partial derivatives. Direct computation gives the following.

$$
\begin{aligned}
\frac{\partial z_{0}}{\partial x}(x, y)= & \frac{1}{\Delta x_{i}}\left(-6 r+6 r^{2}+3 s^{2}\right) z_{i \jmath}+\left(1-4 r+3 r^{2}+\frac{1}{2} s^{2}\right) z_{\imath \jmath}^{x}+\frac{\Delta y_{j}}{\Delta x_{i}}\left(-s+s^{2}\right) z_{i \jmath}^{y} \\
& +\frac{1}{\Delta x_{i}}\left(6 r-6 r^{2}-3 s^{2}\right) z_{i+1, \jmath}+\left(-2 r+3 r^{2}+\frac{1}{2} s^{2}\right) z_{\imath+1, \jmath}^{x}+\frac{\Delta y_{j}}{\Delta x_{\imath}}\left(s-s^{2}\right) z_{\imath+1,3}^{y} \\
& +\frac{1}{\Delta x_{\imath}}\left(-3 s^{2}\right) z_{i, j+1}+\left(-\frac{1}{2} s^{2}\right) z_{i, j+1}^{x}+\frac{\Delta y_{j}}{\Delta x_{i}}\left(s^{2}\right) z_{i, j+1}^{y} \\
& +\frac{1}{\Delta x_{\imath}}\left(3 s^{2}\right) z_{i+1, \jmath+1}+\left(-\frac{1}{2} s^{2}\right) z_{i+1, \jmath+1}^{x}+\frac{\Delta y_{j}}{\Delta x_{i}}\left(-s^{2}\right) z_{i+1, \jmath+1}^{y}
\end{aligned}
$$

$$
\begin{aligned}
& \frac{\partial z_{0}}{\partial y}(x, y)=\frac{1}{\Delta y_{j}}\left(-6 s+6 r s+3 s^{2}\right) z_{i j}+\frac{\Delta x_{i}}{\Delta y_{j}}(-s+r s) z_{\imath j}^{x}+\left(1-r-3 s+2 r s+\frac{3}{2} s^{2}\right) z_{i j}^{y} \\
& +\frac{1}{\Delta y_{j}}\left(-6 r s+3 s^{2}\right) z_{i+1,3}+\frac{\Delta x_{2}}{\Delta y_{3}}(r s) z_{i+1, \jmath}^{x}+\left(r-s-2 r s+\frac{3}{2} s^{2}\right) z_{i+1,3}^{y} \\
& +\frac{1}{\Delta y_{\jmath}}\left(6 s-6 r s-3 s^{2}\right) z_{i, 3+1}+\frac{\Delta x_{2}}{\Delta y_{3}}(s-r s) z_{i, 3+1}^{x}+\left(-2 s+2 r s+\frac{3}{2} s^{2}\right) z_{i, j+1}^{y} \\
& +\frac{1}{\Delta y_{\jmath}}\left(6 r s-3 s^{2}\right) z_{i+1, \jmath+1}+\frac{\Delta x_{2}}{\Delta y_{\jmath}}(-r s) z_{i+1, \jmath+1}^{x}+\left(-2 r s+\frac{3}{2} s^{2}\right) z_{i+1, \jmath+1}^{y}
\end{aligned}
$$

$$
\begin{aligned}
& \frac{\partial z_{1}}{\partial x}(x, y)=\frac{1}{\Delta x_{i}}\left(3(1-r)^{2}-6(1-r)(1-s)\right) z_{i \jmath}+\left(\frac{3}{2}(1-r)^{2}-2(1-r)(1-s)\right) z_{\imath \jmath}^{x} \\
& +\frac{\Delta y_{j}}{\Delta x_{2}}(-(1-r)(1-s)) z_{i j}^{y}+\frac{1}{\Delta x_{i}}\left(-3(1-r)^{2}+6(1-r)(1-s)\right) z_{i+1,3} \\
& +\left(-(1-r)+\frac{3}{2}(1-r)^{2}+(1-s)-2(1-r)(1-s)\right) z_{i+1,3}^{x}+\frac{\Delta y_{j}}{\Delta x_{i}}((1-r)(1-s)) z_{\imath+1,3}^{y} \\
& +\frac{1}{\Delta x_{i}}\left(-6(1-r)+3(1-r)^{2}+6(1-r)(1-s)\right) z_{i, \jmath+1} \\
& +\left(-2(1-r)+\frac{3}{2}(1-r)^{2}+2(1-r)(1-s)\right) z_{i, j+1}^{x}+\frac{\Delta y_{j}}{\Delta x_{i}}((1-r)-(1-r)(1-s)) z_{i, j+1}^{y} \\
& +\frac{1}{\Delta x_{i}}\left(6(1-r)-3(1-r)^{2}-6(1-r)(1-s)\right) z_{i+1, \jmath+1} \\
& +\left(1-3(1-r)+\frac{3}{2}(1-r)^{2}-(1-s)+2(1-r)(1-s)\right) z_{2+1,3+1}^{r} \\
& +\frac{\Delta y_{3}}{\Delta x_{2}}(-(1-r)+(1-r)(1-s)) z_{i+1, \jmath+1}^{y}
\end{aligned}
$$$$
\frac{\partial z_{1}}{\partial y}(x, y)=\frac{1}{\Delta y_{j}}\left(-3(1-r)^{2}\right) z_{i j}+\frac{\Delta x_{i}}{\Delta y_{j}}\left(-(1-r)^{2}\right) z_{i j}^{x}+\left(-\frac{1}{2}(1-r)^{2}\right) z_{i j}^{y}
$$$$
+\frac{1}{\Delta y_{j}}\left(3(1-r)^{2}-6(1-s)+6(1-s)^{2}\right) z_{i+1, j}+\frac{\Delta x_{2}}{\Delta y_{3}}\left((1-r)-(1-r)^{2}\right) z_{i+1, j}^{x}
$$$$
+\left(\frac{1}{2}(1-r)^{2}-2(1-s)+3(1-s)^{2}\right) z_{2+1, j}^{y}+\frac{1}{\Delta y_{j}}\left(3(1-r)^{2}\right) z_{i, j+1}
$$ 


$$
\begin{aligned}
& +\frac{\Delta x_{i}}{\Delta y_{j}}\left((1-r)^{2}\right) z_{i, j+1}^{x}+\left(-\frac{1}{2}(1-r)^{2}\right) z_{i, j+1}^{y} \\
& +\frac{1}{\Delta y_{j}}\left(-3(1-r)^{2}+6(1-s)-6(1-s)^{2}\right) z_{i+1, j+1}+\frac{\Delta x_{i}}{\Delta y_{j}}\left(-(1-r)+(1-r)^{2}\right) z_{i+1, j+1}^{x} \\
& +\left(1+\frac{1}{2}(1-r)^{2}-4(1-s)+3(1-s)^{2}\right) z_{i+1, j+1}^{y} \\
& \frac{\partial z_{2}}{\partial x}(x, y)=\frac{1}{\Delta x_{i}}\left(-3(1-r)^{2}\right) z_{i j}+\left(-\frac{1}{2}(1-r)^{2}\right) z_{i j}^{x}+\frac{\Delta y_{j}}{\Delta x_{i}}\left(-(1-r)^{2}\right) z_{i j}^{y} \\
& +\frac{1}{\Delta x_{i}}\left(3(1-r)^{2}\right) z_{i+1, j}+\left(-\frac{1}{2}(1-r)^{2}\right) z_{i+1, j}^{x}+\frac{\Delta y_{j}}{\Delta x_{i}}\left((1-r)^{2}\right) z_{i+1, j}^{y} \\
& +\frac{1}{\Delta x_{i}}\left(-6(1-r)+6(1-r)^{2}+3(1-s)^{2}\right) z_{i, j+1}+\left(-2(1-r)+3(1-r)^{2}+\frac{1}{2}(1-s)^{2}\right) z_{i, j+1}^{x} \\
& +\frac{\Delta y_{j}}{\Delta x_{i}}\left((1-r)-(1-r)^{2}\right) z_{i, j+1}^{y}+\frac{1}{\Delta x_{i}}\left(6(1-r)-6(1-r)^{2}-3(1-s)^{2}\right) z_{i+1, j+1} \\
& +\left(1-4(1-r)+3(1-r)^{2}+\frac{1}{2}(1-s)^{2}\right) z_{i+1, j+1}^{x}+\frac{\Delta y_{j}}{\Delta x_{i}}\left(-(1-r)+(1-r)^{2}\right) z_{i+1, j+1}^{y} \\
& \frac{\partial z_{2}}{\partial y}(x, y)=\frac{1}{\Delta y_{j}}\left(-6(1-r)(1-s)+3(1-r)^{2}\right) z_{i j}+\frac{\Delta x_{i}}{\Delta y_{j}}(-(1-r)(1-s)) z_{i j}^{x} \\
& +\left(-2(1-r)(1-s)+\frac{3}{2}(1-s)^{2}\right) z_{i j}^{y}+\frac{1}{\Delta y_{j}}\left(-6(1-s)+6(1-r)(1-s)+3(1-s)^{2}\right) z_{i+1, j} \\
& +\frac{\Delta x_{i}}{\Delta y_{j}}((1-s)-(1-r)(1-s)) z_{i+1, j}^{x}+\left(-2(1-s)+2(1-r)(1-s)+\frac{3}{2}(1-s)^{2}\right) z_{i+1, j}^{y} \\
& +\frac{1}{\Delta y_{j}}\left(6(1-r)(1-s)-3(1-s)^{2}\right) z_{i, j+1}+\frac{\Delta x_{i}}{\Delta y_{j}}((1-r)(1-s)) z_{i, j+1}^{x} \\
& +\left((1-r)-(1-s)-2(1-r)(1-s)+\frac{3}{2}(1-s)^{2}\right) z_{i, j+1}^{y} \\
& +\frac{1}{\Delta y_{j}}\left(+6(1-r)-6(1-r)(1-s)-3(1-s)^{2}\right) z_{i+1, j+1} \\
& +\frac{\Delta x_{i}}{\Delta y_{j}}(-(1-s)+(1-r)(1-s)) z_{i+1, j+1}^{x} \\
& +\left(1-(1-r)-3(1-s)+(1-r)(1-s)+\frac{3}{2}(1-s)^{2}\right) z_{i+1, j+1}^{y} \\
& \frac{\partial z_{3}}{\partial x}(x, y)=\frac{1}{\Delta x_{i}}\left(-6 r+3 r^{2}+6 r s\right) z_{i j}+\left(1-3 r+\frac{3}{2} r^{2}-s+2 r s\right) z_{i j}^{x}+\frac{\Delta y_{j}}{\Delta x_{i}}(-r+r s) z_{i j}^{y} \\
& +\frac{1}{\Delta x_{i}}\left(6 r-3 r^{2}-6 r s\right) z_{i+1, j}+\left(-2 r+\frac{3}{2} r^{2}+2 r s\right) z_{i+1, j}^{x} \\
& +\frac{\Delta y_{j}}{\Delta x_{i}}(r-r s) z_{i+1, j}^{y}+\frac{1}{\Delta x_{i}}\left(3 r^{2}-6 r s\right) z_{i, j+1} \\
& +\left(-r+\frac{3}{2} r^{2}+s-2 r s\right) z_{i, j+1}^{x}+\frac{\Delta y_{j}}{\Delta x_{i}}(r s) z_{i, j+1}^{y} \\
& +\frac{1}{\Delta x_{i}}\left(-3 r^{2}+6 r s\right) z_{i+1, j+1}+\left(\frac{3}{2} r^{2}-2 r s\right) z_{i+1, j+1}^{x}+\frac{\Delta y_{j}}{\Delta x_{i}}(-r s) z_{i+1, j+1}^{y}
\end{aligned}
$$




$$
\begin{aligned}
\frac{\partial z_{3}}{\partial y}(x, y)= & \frac{1}{\Delta y_{j}}\left(3 r^{2}-6 s+6 s^{2}\right) z_{i j}+\frac{\Delta x_{i}}{\Delta y_{j}}\left(-r+r^{2}\right) z_{i j}^{x} \\
& +\left(1+\frac{1}{2} r^{2}-4 s+3 s^{2}\right) z_{i j}^{y}+\frac{1}{\Delta y_{j}}\left(-3 r^{2}\right) z_{i+1, j}+\frac{\Delta x_{i}}{\Delta y_{j}}\left(r^{2}\right) z_{i+1, j}^{x} \\
& +\left(-\frac{1}{2} r^{2}\right) z_{i+1,3}^{y}+\frac{1}{\Delta y_{j}}\left(-3 r^{2}+6 s-6 s^{2}\right) z_{i, j+1} \\
& +\frac{\Delta x_{i}}{\Delta y_{j}}\left(r-r^{2}\right) z_{i, j+1}^{x}+\left(\frac{1}{2} r^{2}-2 s+3 s^{2}\right) z_{i, j+1}^{y}+\frac{1}{\Delta y_{j}}\left(3 r^{2}\right) z_{i+1, j+1} \\
& +\frac{\Delta x_{i}}{\Delta y_{j}}\left(-r^{2}\right) z_{i+1, j+1}^{x}+\left(-\frac{1}{2} r^{2}\right) z_{i+1, j+1}^{y}
\end{aligned}
$$

\subsection{Second Derivatives}

In this section only those terms with nonzero coefficients will be written out. Thus all coefficients of corner data items that are zero will not be included in the second derivative expansions.

$$
\begin{aligned}
& \frac{\partial^{2} z_{0}}{\partial x^{2}}(x, y)=\frac{1}{\Delta x_{i}^{2}}(-6+12 r) z_{i j}+\frac{1}{\Delta x_{i}}(-4+6 r) z_{i j}^{x} \\
& +\frac{1}{\Delta x_{i}^{2}}(6-12 r) z_{i+1, j}+\frac{1}{\Delta x_{i}}(-2+6 r) z_{i+1, j}^{x} \\
& \frac{\partial^{2} z_{0}}{\partial y \partial x}(x, y)=\frac{1}{\Delta x_{i} \Delta y_{\jmath}}(6 s) z_{i j}+\frac{1}{\Delta y_{j}}(s) z_{i j}^{x}+\frac{1}{\Delta x_{i}}(-1+2 s) z_{i j}^{y} \\
& +\frac{1}{\Delta x_{2} \Delta y_{j}}(-6 s) z_{i+1, j}+\frac{1}{\Delta y_{j}}(s) z_{i+1, j}^{x}+\frac{1}{\Delta x_{i}}(1-2 s) z_{i+1, j}^{y} \\
& +\frac{1}{\Delta x_{i} \Delta y_{j}}(-6 s) z_{i, j+1}+\frac{1}{\Delta y_{j}}(-s) z_{i, \jmath+1}^{x} \\
& +\frac{1}{\Delta x_{i}}(2 s) z_{i, j+1}^{y}+\frac{1}{\Delta x_{i} \Delta y_{j}}(6 s) z_{i+1, j+1} \\
& +\frac{1}{\Delta y_{j}}(-s) z_{i+1, j+1}^{x}+\frac{1}{\Delta x_{i}}(-2 s) z_{i, j+1}^{y} \\
& \frac{\partial^{2} z_{0}}{\partial y^{2}}(x, y)=\frac{1}{\Delta y_{j}^{2}}(-6+6 r+6 s) z_{i j}+\frac{\Delta x_{i}}{\Delta y_{3}^{2}}(-1+r) z_{i j}^{x} \\
& +\frac{1}{\Delta y_{j}}(-3+2 r+3 s) z_{i j}^{y}+\frac{1}{\Delta y_{j}^{2}}(-6 r+6 s) z_{i+1, j} \\
& +\frac{\Delta x_{i}}{\Delta y_{j}^{2}}(r) z_{i+1, j}^{x}+\frac{1}{\Delta y_{j}}(-1-2 r+3 s) z_{i+1, j}^{y} \\
& +\frac{1}{\Delta y_{j}^{2}}(6-6 r-6 s) z_{i, \jmath+1}+\frac{\Delta x_{i}}{\Delta y_{3}^{2}}(1-r) z_{i, j+1}^{x} \\
& +\frac{1}{\Delta y_{j}}(-2+2 r+3 s) z_{i, j+1}^{y}+\frac{1}{\Delta y_{j}^{2}}(6 r-6 s) z_{i+1, \jmath+1} \\
& +\frac{\Delta x_{i}}{\Delta y_{j}^{2}}(-r) z_{i+1, j+1}^{x}+\frac{1}{\Delta y_{j}}(-2 r+3 s) z_{i+1, j+1}^{y}
\end{aligned}
$$




$$
\begin{aligned}
& \frac{\partial^{2} z_{1}}{\partial x^{2}}(x, y)=\frac{1}{\Delta x_{i}^{2}}(-6(1-r)+6(1-s)) z_{i j}+\frac{1}{\Delta x_{\imath}}(-3(1-r)+2(1-s)) z_{i j}^{x} \\
& +\frac{\Delta y_{j}}{\Delta x_{i}^{2}}((1-s)) z_{i j}^{y}+\frac{1}{\Delta x_{i}^{2}}(6(1-r)-6(1-s)) z_{i+1,3} \\
& +\frac{1}{\Delta x_{i}}(1-3(1-r)+2(1-s)) z_{i+1, j}^{x}+\frac{\Delta y_{j}}{\Delta x_{i}^{2}}(-(1-s)) z_{i+1, j}^{y} \\
& +\frac{1}{\Delta x_{i}^{2}}(6-6(1-r)-6(1-s)) z_{i, j+1}+\frac{1}{\Delta x_{i}}(2-3(1-r)-2(1-s)) z_{i, j+1}^{x} \\
& +\frac{\Delta y_{j}}{\Delta x_{i}^{2}}(-1+(1-s)) z_{i, j+1}^{y}+\frac{1}{\Delta x_{i}^{2}}(-6+6(1-r)+6(1-s)) z_{i+1, j+1} \\
& +\frac{1}{\Delta x_{i}}(3-3(1-r)-2(1-s)) z_{i+1, \jmath+1}^{x}+\frac{\Delta y_{j}}{\Delta x_{i}^{2}}(1-(1-s)) z_{i+1, j+1}^{y} \\
& \frac{\partial^{2} z_{1}}{\partial y \partial x}(x, y)=\frac{1}{\Delta x_{i} \Delta y_{j}}(6(1-s)) z_{i j}+\frac{1}{\Delta y_{j}}(2(1-s)) z_{i j}^{x}+\frac{1}{\Delta x_{i}}((1-r)) z_{i j}^{y} \\
& +\frac{1}{\Delta x_{i} \Delta y_{j}}(-6(1-s)) z_{i+1, j}+\frac{1}{\Delta y_{j}}(-1+2(1-r)) z_{i+1, j}^{x}+\frac{1}{\Delta x_{i}}(-(1-r)) z_{i+1, j}^{y} \\
& +\frac{1}{\Delta x_{i} \Delta y_{j}}(-6(1-r)) z_{i, j+1}+\frac{1}{\Delta y_{j}}(-2(1-r)) z_{i, j+1}^{x}+\frac{1}{\Delta x_{i}}((1-r)) z_{i, j+1}^{y} \\
& +\frac{1}{\Delta x_{i} \Delta y_{j}}(6(1-r)) z_{i+1, j+1}+\frac{1}{\Delta y_{j}}(1-2(1-r)) z_{i+1, j+1}^{x}+\frac{1}{\Delta x_{i}}(-(1-r)) z_{i, j+1}^{y} \\
& \frac{\partial^{2} z_{1}}{\partial y^{2}}(x, y)=\frac{1}{\Delta y_{j}^{2}}(6-12(1-s)) z_{i+1, j} \frac{1}{\Delta y_{j}}(2-6(1-s)) z_{i+1, j}^{y} \\
& \frac{1}{\Delta y_{\jmath}^{2}}(-6+12(1-s)) z_{i+1, j+1} \frac{1}{\Delta y_{3}}(4-6(1-s)) z_{i+1, j+1}^{y} \\
& \frac{\partial^{2} z_{2}}{\partial x^{2}}(x, y)=\frac{1}{\Delta x_{i}^{2}}(6-12(1-r)) z_{i, j+1} \frac{1}{\Delta x_{i}}(2-6(1-r)) z_{i, j+1}^{x} \\
& \frac{1}{\Delta x_{i}^{2}}(-6+12(1-r)) z_{\imath+1, j+1} \frac{1}{\Delta x_{i}}(4-6(1-r)) z_{i+1, j+1}^{x} \\
& \frac{\partial^{2} z_{2}}{\partial y \partial x}(x, y)=\frac{1}{\Delta x_{i} \Delta y_{j}}(6(1-s)) z_{i j}+\frac{1}{\Delta y_{j}}((1-s)) z_{i j}^{x}+\frac{1}{\Delta x_{i}}(2(1-s)) z_{i j}^{y} \\
& +\frac{1}{\Delta x_{i} \Delta y_{j}}(-6(1-s)) z_{i+1,3}+\frac{1}{\Delta y_{j}}((1-s)) z_{i+1,3}^{x}+\frac{1}{\Delta x_{i}}(-2(1-s)) z_{i+1,3}^{y} \\
& +\frac{1}{\Delta x_{2} \Delta y_{j}}(-6(1-s)) z_{i, j+1}+\frac{1}{\Delta y_{j}}(-(1-s)) z_{i, j+1}^{x}+\frac{1}{\Delta x_{i}}(-1+2(1-s)) z_{i, j+1}^{y} \\
& +\frac{1}{\Delta x_{\imath} \Delta y_{j}}(6(1-s)) z_{i+1, j+1}+\frac{1}{\Delta y_{j}}(-(1-s)) z_{i+1, j+1}^{x}+\frac{1}{\Delta x_{i}}(1-2(1-s)) z_{i, j+1}^{y} \\
& \frac{\partial^{2} z_{2}}{\partial y^{2}}(x, y)=\frac{1}{\Delta y_{j}^{2}}(6(1-r)-6(1-s)) z_{i j}+\frac{\Delta x_{i}}{\Delta y_{j}^{2}}((1-r)) z_{i j}^{x} \\
& +\frac{1}{\Delta y_{3}}(2(1-r)-3(1-s)) z_{i j}^{y}+\frac{1}{\Delta y_{3}^{2}}(6-6(1-r)-6(1-s)) z_{i+1, j} \\
& +\frac{\Delta x_{i}}{\Delta y_{j}^{2}}(-1+(1-s)) z_{i+1, j}^{x}+\frac{1}{\Delta y_{j}}(2-2(1-r)-3(1-s)) z_{i+1, j}^{y}
\end{aligned}
$$




$$
\begin{aligned}
& +\frac{1}{\Delta y_{j}^{2}}(-6(1-r)+6(1-s)) z_{i, \jmath+1}+\frac{\Delta x_{i}}{\Delta y_{j}^{2}}(-(1-r)) z_{i, j+1}^{x} \\
& +\frac{1}{\Delta y_{i}}(1+2(1-r)-3(1-s)) z_{i, j+1}^{y}+\frac{1}{\Delta y_{j}^{2}}(-6+6(1-r)+6(1-s)) z_{i+1, j+1} \\
& +\frac{\Delta x_{i}}{\Delta y_{j}^{2}}(1-(1-r)) z_{i+1, j+1}^{x}+\frac{1}{\Delta y_{j}}(3-2(1-r)-3(1-s)) z_{i+1, \jmath+1}^{y} \\
& \frac{\partial^{2} z_{3}}{\partial x^{2}}(x, y)=\frac{1}{\Delta x_{i}^{2}}(-6+6 r+6 s) z_{i j}+\frac{1}{\Delta x_{i}}(-3+3 r+2 s) z_{i j}^{x}+\frac{\Delta y_{j}}{\Delta x_{i}^{2}}(-1+s) z_{i j}^{y} \\
& +\frac{1}{\Delta x_{i}^{2}}(6-6 r-6 s) z_{i+1, j}+\frac{1}{\Delta x_{i}}(-2+3 r+2 s) z_{i+1, j}^{x}+\frac{\Delta y_{j}}{\Delta x_{i}^{2}}((1-s)) z_{i+1, j}^{y} \\
& +\frac{1}{\Delta x_{i}^{2}}(6 r-6 s) z_{i, j+1}+\frac{1}{\Delta x_{i}}(-1+3 r-2 s) z_{i, j+1}^{x}+\frac{\Delta y_{j}}{\Delta x_{i}^{2}}(s) z_{i, j+1}^{y} \\
& +\frac{1}{\Delta x_{i}^{2}}(-6 r+6 s) z_{i+1, j+1}+\frac{1}{\Delta x_{i}}(3 r-2 s) z_{i+1, j+1}^{x}+\frac{\Delta y_{j}}{\Delta x_{i}^{2}}(-s) z_{i+1, j+1}^{y} \\
& \frac{\partial^{2} z_{3}}{\partial y \partial x}(x, y)=\frac{1}{\Delta x_{\imath} \Delta y_{j}}(6 r) z_{i j}+\frac{1}{\Delta y_{j}}(-1+2 r) z_{i j}^{x}+\frac{1}{\Delta x_{i}}(r) z_{i j}^{y} \\
& +\frac{1}{\Delta x_{i} \Delta y_{j}}(-6 r) z_{i+\mathbf{1}, j}+\frac{1}{\Delta y_{j}}(2 r) z_{i+1, j}^{x}+\frac{1}{\Delta x_{i}}(-r) z_{i+\mathbf{1}, j}^{y} \\
& +\frac{1}{\Delta x_{i} \Delta y_{j}}(-6 r) z_{i, j+1}+\frac{1}{\Delta y_{j}}(1-2 r) z_{i, j+1}^{x}+\frac{1}{\Delta x_{i}}(r) z_{i, j+1}^{y} \\
& +\frac{1}{\Delta x_{i} \Delta y_{j}}(6 r) z_{i+1, \jmath+1}+\frac{1}{\Delta y_{j}}(-2 r) z_{i+1, j+1}^{x}+\frac{1}{\Delta x_{i}}(-r) z_{i, j+1}^{y} \\
& \frac{\partial^{2} z_{3}}{\partial y^{2}}(x, y)=\frac{1}{\Delta y_{j}^{2}}(-6+12 s) z_{i j}+\frac{1}{\Delta y_{j}}(-4+6 s) z_{i j}^{y} \\
& +\frac{1}{\Delta y_{j}^{2}}(6-12 s) z_{i, j+1}+\frac{1}{\Delta y_{j}}(-2+6 s) z_{i, j+1}^{y}
\end{aligned}
$$

\section{References}

[1] Han, L. and Schumaker, L. L., Fitting monotone surfaces to scattered data using $C_{1}$ piecewise cubics. SIAM J. Numer. Anal. 34 (1997), 569-585.

[2] Lavery, J. E., Univariate cubic $L_{p}$ splines and shape preserving, multiscale interpolation by univariate cubic $L_{\mathbf{1}}$ splines. Comput. Aided Geom. Design 17 (2000), 319-336.

[3] Lavery, J. E., Shape-preserving, multiscale fitting of univariate data by cubic $L_{1}$ smoothing splines. Comput. Aided Geom. Design 17 (2000), 715-727.

[4] Lavery, J. E., Shape-preserving, multiscale interpolation by bi- and multivariate cubic $L_{1}$ splines. Comput. Aided Geom. Design To appear.

[5] Lawson, C. L., $C^{1}$-compatible interpolation over a triangle. Technical Memorandum No. 33-770, Jet Propulsion Laboratory, Pasadena, California, 1976.

[6] Lawson, C. L., Software for $C^{1}$ surface interpolation. In Mathematical Software III, Proceedings of a Symposium conducted by the Mathematics Research Center, University of Wisconsin, Madison (New York, 1977), J. R. Rice, Ed., Academic Press, pp. 161-194. 


